\title{
Quelle recherche en didactique pour la filière Langues étrangères appliquées?
}

\section{Marie-Françoise Narcy-Combes}

\section{(2) OpenEdition}

\section{Journals}

Édition électronique

URL : http://journals.openedition.org/asp/4510

DOI : 10.4000/asp.4510

ISBN : 978-2-8218-0398-5

ISSN : 2108-6354

Éditeur

Groupe d'étude et de recherche en anglais de spécialité

Édition imprimée

Date de publication : 1 décembre 2004

Pagination : 155-163

ISSN : 1246-8185

\section{Référence électronique}

Marie-Françoise Narcy-Combes, « Quelle recherche en didactique pour la filière Langues étrangères appliquées ? », ASp [En ligne], 45-46 | 2004, mis en ligne le 04 août 2014, consulté le 01 mai 2019.

URL : http://journals.openedition.org/asp/4510 ; DOI : 10.4000/asp.4510

Ce document a été généré automatiquement le 1 mai 2019.

Tous droits réservés 


\title{
Quelle recherche en didactique pour la filière Langues étrangères appliquées?
}

\author{
Marie-Françoise Narcy-Combes
}

\section{Introduction}

1 Cet article n'est pas un article de recherche en tant que tel, mais un appel argumenté et théorisé pour le développement d'un groupe de travail, qui a pris appui sur des données scientifiques solides. Il s'inscrit dans un mouvement fondateur pour la recherche didactique en Langues étrangères appliquées (LEA) impulsé depuis mars 2004 et fait suite à l'atelier de réflexion sur ce thème au colloque du GERAS de mars 2005 à Toulouse. L'objectif est d'en développer la dynamique. En mars 2004 à Poitiers, au cous du colloque précédent, les enseignants-chercheurs réunis en atelier remarquaient qu'il était nécessaire d'une part de renforcer le niveau en langue des étudiants et de développer leur autonomie, d'autre part de travailler à la cohérence des contenus au sein d'une même langue entre enseignement culturel, traduction et langue de spécialité, entre les différentes langues et entre les langues et les matières d'application. Il s'agit bien de proposer une recherche sur l'apprentissage des contenus, distincte de celle déjà existante sur les contenus eux-mêmes. En effet, la recherche en langue de spécialité ou en civilisation et en interculturel, si elle alimente le travail sur les contenus, n'est pas spécifique à une filière d'enseignement. Or, force est de constater que traditionnellement, en France, on valorise les contenus plutôt que la manière dont on peut les acquérir (voir à ce propos les travaux en sociologie de l'éducation). La recherche dans les domaines de langue de spécialité ou culturels ne peut suffire à assurer l'essor d'une filière d'enseignement, de LEA en ce qui nous concerne, car il reste à gérer les dispositifs institutionnels et les acquisitions individuelles qui ne sont pas nécessairement pris en compte par les chercheurs des disciplines citées.

2 Les chercheurs réunis à Poitiers se proposaient par ailleurs de travailler en séminaires localement, et de proposer l'organisation d'une journée d'étude. Ces éléments relèvent du 
débat classique en LEA. S'il perdure, c'est bien que la recherche n'a pas apporté de solution satisfaisante jusque-là. Un an plus tard, le bilan nous oblige à constater une fois de plus le manque crucial de motivation des enseignants-chercheurs impliqués dans la filière. Certes, la recherche en LEA ne saurait se limiter à une recherche en didactique, mais lorsqu'elle n'est pas didactique, les chercheurs ne mènent pas de recherche spécifique LEA et rejoignent des équipes plus prestigieuses. Il serait d'ailleurs intéressant de lancer une enquête socioprofessionnelle qui pourrait nous apporter des éléments essentiels sur ce point. C'est pourquoi cet article commence par une profession de foi sur la nécessité de développer un domaine de recherche sur lequel les enseignements pourraient s'appuyer. Pour le moment, celui-ci bénéficie de recherches du secteur LANSAD $^{1}$, mais si l'on postule qu'il ne peut y avoir de recherche en sciences humaines sans prise en compte du contexte, on peut dire que la recherche spécifique à la filière LEA reste à définir. En effet, nous avons affaire à des étudiants qui ne sont ni des spécialistes de langues à proprement parler, ni des spécialistes d'autres disciplines, ce que sont les étudiants LANSAD. À la suite de G. Simondon pour lequel il existe une relation transductive entre Homme, technologie et société, J.-P. Narcy-Combes (2005) postule qu'il $\mathrm{y}$ a le même type de relation entre langue, savoir(s) et culture. Le problème que rencontrent les étudiants de LEA, c'est qu'ils n'ont pas de spécialité disciplinaire. Arrivés en Master 2, les étudiants en Langue, littérature et civilisation étrangères (LLCE) sont spécialisés en littérature, civilisation ou linguistique. Ce n'est pas le cas des étudiants de LEA, bien qu'ils soient eux aussi considérés comme spécialistes. Ils n'ont donc pas de raison de se servir de la langue. Il y a là en germe une question de recherche sur les effets que cette relation peut avoir sur eux. En d'autres termes, les étudiants de LEA, nous l'avons maintes fois constaté, ne se sentent légitimes en rien, car ils ne sont spécialistes ni en langues, ni dans les matières d'application: quel effet cela peut-il avoir sur leurs apprentissages?

D'autre part, leur point fort et leur potentiel résident dans un plurilinguisme qui s'appuie sur des contenus variés, un contexte qui semble se rattacher aux théories qui soustendent ce qu'on appelle Content and Language Integrated Learning. Or ces deux termes posent problème. Dans le sens que le Conseil de l'Europe donne à ces termes, l'enseignement en LEA n'est pas à proprement parler plurilingue. En effet, il n'y a pas appui d'une langue sur l'autre, dans la mesure où chaque langue est un champ séparé des autres. Il y a, là encore, un vaste domaine de recherche non exploré. D'autre part, pour que l'enseignement s'appuie sur un contenu, il faudrait que les étudiants soient spécialistes du contenu, ce qu'ils ne semblent pas être. Un autre terrain d'enquête s'ouvre ici : lorsque ces étudiants sont employés par l'entreprise à la sortie de leurs études, le sont-ils en tant que spécialistes de langues ou comme spécialistes d'une autre discipline?

Enfin, LEA est souvent une filière où l'on s'inscrit par défaut (Narcy-Combes 2003). On peut alors se demander s'il est pertinent d'appliquer aux étudiants qui s'y retrouvent les traditions d'excellence dont la France se fait une spécialité. Au vu des performances des étudiants, peut-on ne pas s'interroger sur l'organisation des apprentissages davantage encore que sur les contenus? Peut-on véritablement occulter le fait que les autres filières professionnalisantes tels que les BTS, les IUT, les écoles de commerce et d'ingénieurs, fonctionnent avec de petits nombres, alors que leur recrutement est sélectif, tandis que les LEA fonctionnent avec de grands nombres, alors même que le recrutement y est moins axé sur les performances? Peut-on vraiment transférer ce qui se fait ailleurs sans réflexion didactique? 
5 Ces éléments constituent la spécificité du contexte et engendrent une série de contraintes qu'il serait nécessaire de déterminer, et d'analyser, de façon à pouvoir envisager la mise en place de tâches et de dispositifs en fonction des données théoriques actuelles en ce qui concerne les apprentissages langagiers. Rien ne dit d'ailleurs qu'un certain nombre de ces contraintes ne sont pas antinomiques avec les théories. Mesurer ce qui se fait actuellement dans cette filière, notamment en ce qui concerne l'enseignement culturel ou celui de la traduction, et confronter ces pratiques aux théories paraît être une démarche indispensable pour répondre aux besoins $\mathrm{du}$ nombre en constante augmentation d'étudiants qui s'y engagent.

6 Après ce positionnement, nous nous proposons d'ouvrir quelques pistes de recherche à explorer. Elles concernent le contexte d'enseignement/apprentissage, incluant les caractéristiques du public des intervenants de LEA, les contenus d'enseignement adaptés à ce public et à ses objectifs, et les dispositifs en place et à inventer pour mettre en œuvre l'apprentissage.

7 Comme l'a rappelé David Little (2005) au cours de la conférence plénière du colloque de Toulouse, la mise en place d'un enseignement/apprentissage en langue étrangère devrait avoir pour objectif de rendre les apprenants autonomes, capables d'acquérir et de développer des savoirs et savoir-faire langagiers tout au long de leur vie. Ceci devrait conduire les décideurs à s'interroger sur les contenus et les dispositifs à proposer, et également sur les modes d'acquisition des contenus dans les dispositifs par la population étudiante spécifique à ces dispositifs, et au préalable connaître l'objectif à atteindre et le potentiel des apprenants pour les atteindre. La recherche sur les dispositifs et les modes d'acquisition relève de la didactique si les contenus relèvent d'autres domaines.

8 En termes d'objectifs spécifiques à la filière, le groupe de travail du GERAS réuni en 2004 avait proposé que les enseignants de LEA s'entendent sur un niveau minimal commun à atteindre en langues pour tous les étudiants de la fi ère. Il conviendrait que ce seuil soit réaliste et, à cet effet, D. Little soulignait que les niveaux européens B1 en production et A2 en réception correspondraient mieux à la réalité du terrain que les ambitions des décideurs dans la filière ne le laissent supposer. Réfléchir en ces termes permettrait d'aller plus loin dans la cohérence et l'harmonisation des parcours que le simple fait de fixer un niveau plancher pour les langues comme cela se pratique dans plusieurs universités, à Grenoble en particulier où les enseignants se montrent très attachés à cette pratique.

9 Pour initier ce travail, il serait nécessaire de mener une recherche-action d'envergure, ce qui ne saurait être possible sans la création d'une culture commune au groupe de recherche constitué. C'est la condition première pour constituer un programme de recherche. Un programme en recherche-action ne peut être autre qu'un cadre méthodologique et organisationnel, d'autant que la culture de groupe n'est pas mise en place. Il ne saurait être déterminé de manière rigoureuse en raison des spécificités et de la complexité du contexte. C'est en cela que nous nous plaçons dans le cadre de la pensée complexe (Morin 2005). Ce positionnement permet de mieux fonctionner dans le contexte mouvant de l'enseignement/apprentissage étant donné la multiplicité des paramètres qui entrent en jeu, des systèmes qui interagissent, de l'imprévisibilité et de la mouvance de chacun. Il n'est pas envisageable alors de penser pouvoir prendre des décisions parfaites, univoques et définitives. 


\section{Le contexte socioculturel}

Les étudiants, les enseignants et l'institution forment chacun des systèmes complexes qui interagissent et dont il est difficile de maîtriser tous les paramètres. La complexité du contexte de la filière LEA mériterait que la recherche intègre le travail de sociologues de l'éducation comme François Dubet et d'économistes de l'éducation comme Éric Delamotte.

\subsection{Les étudiants}

11 En ce qui concerne le public, plusieurs constats ont été faits qui demandent à être vérifiés. Ils suscitent des hypothèses qui doivent être validées. Une première série d'interrogations concerne l'investissement des étudiants. Le travail qualitatif effectué par deux étudiantes en sociologie de l'université Paris $7^{2}$ fournit des pistes concernant le décalage entre le sentiment qu'ont les professeurs que les étudiants échouent dans cette filière, et les perceptions des étudiants qui n'ont pas le même point de vue. En effet, certains étudiants entrent en LEA pour ne pas faire de choix tout de suite après le baccalauréat et viennent là pour attendre ou pour une autre raison que celle de se former dans une spécialité liée aux études en LEA. L'enquête ne concernait que 10 enseignants et 20 étudiants en difficulté. Cependant, le phénomène a déjà été répertorié par ailleurs et a fait l'objet de nombreux débats, entraînant même un questionnement sur la sélection à l'entrée à l'Université. Ce phénomène n'est pas l'apanage de la filière LEA, et à l'université de Nantes, les chiffres révèlent qu'un fait similaire peut être observé dans d'autres formations, comme le montre le tableau 1.

Tableau 1. Nombre d'étudiants inscrits en LLCE et en LEA à Nantes en 2005

\begin{tabular}{|l|l|l|l|}
\hline & LLCE & & LEA \\
\hline L1 & anglais & 211 & 517 \\
\hline & bilingue & 120 & \\
\hline & bi-disciplinaire & 10 & \\
\hline & Total & 341 & \\
\hline L2 & & 153 & 254 \\
\hline L3 & anglais & 133 & \\
\hline & bilingue & 35 & \\
\hline & Total & 168 & \\
\hline M1 & & 87 & 146 \\
\hline
\end{tabular}



difficile de ne pas s'interroger sur les raisons de la fonte des effectifs que ce tableau fait apparaître. conduisent à s'interroger sur les motivations réelles des étudiants. En effet, dans une filière qui s'intitule "Affaires et commerce international», on s'aperçoit que plus de la moitié des stages ont lieu en France. Si l'on considère les stages qui ont lieu en France, plus des trois quarts ont lieu en Loire-Atlantique. Lorsque les étudiants partent à l'étranger, plus des trois quarts restent dans l'Union européenne. Il serait instructif de comparer ces résultats aux chiffres disponibles dans les autres universités, de façon à pouvoir établir s'il s'agit d'un phénomène généralisé ou d'une caractéristique locale. Cependant, il est d'ores et déjà utile de se demander s'il est positif qu'une forte proportion des étudiants fasse leur stage dans les entreprises de la région. Si ce n'est pas ce que l'on veut, comment faire pour que la situation évolue? On peut se demander si le cas de Nantes est spécifique ou si l'on peut trouver des contextes semblables dans d'autres universités.

Un autre élément semble confirmer cette hypothèse. Depuis le premier semestre universitaire de l'année 2003, j'ai mené une enquête longitudinale avec pour objectif de mesurer l'impact de la formation innovante proposée en maîtrise LEA (NarcyCombes 2003) et la perception que les étudiants ont de la formation en anglais qui leur est proposée. On constate que le taux de participation dans les réponses aux questionnaires est relativement bas (un quart des étudiants environ répondent alors que le questionnaire est distribué en cours). On peut postuler que les étudiants sont peu impliqués et déresponsabilisés. Il est possible également de se demander s'il ne faut pas y voir plutôt l'effet des représentations des étudiants sur l'enseignement/apprentissage : pour eux, ils sont là pour recevoir un savoir transmis par le professeur. On aurait besoin ici de l'apport de la sociologie de l'éducation pour pousser plus avant les investigations.

\subsection{Les enseignants}

Le fait avait déjà été signalé à plusieurs reprises : une convergence d'éléments confirme que peu de professeurs et enseignants-chercheurs HDR s'intéressent vraiment à la filière LEA. Bien sûr, les maîtres de conférences font de la recherche eux aussi, mais ils ne peuvent fonder un laboratoire. Si donc un maitre de conférences s'intéresse à la recherche en didactique pour LEA, il lui faudra se rattacher à un laboratoire extérieur. De ce fait, l'enseignement qui est dispensé n'est rattaché à la recherche que de façon marginale, ce qui ne correspond pas à la vocation de l'université. La filière LEA ne dispose pas d'organe de diffusion de la recherche. L'Association nationale des Langues étrangères appliquées (ANLEA), en tant qu'association, joue un rôle plutôt politique et ne se positionne pas vraiment comme organe de recherche. Il apparaît donc nécessaire que des postes de professeurs soient créés en plus grand nombre en LEA parce qu'il est nécessaire de développer une recherche spécifique à ce domaine. Ce constat pose clairement la question de la motivation des enseignants de la filière, et soulève le problème du recrutement des personnels pour ce département. En effet, les étudiants mériteraient de bénéficier d'un enseignement appuyé sur une recherche spécifique et d'enseignantschercheurs dont les intérêts sont en phase avec leurs besoins en formation. Ce manque 
d'intérêt est par ailleurs en contradiction avec les chiffres croissants d'inscription dans la filière, alors qu'ils diminuent ailleurs.

\section{Les contenus et leur adaptation au public ciblé}

Les échanges de Toulouse ont montré que les écoles d'ingénieurs et les facultés scientifiques sont dans l'ensemble mieux sensibilisées aux exigences de l'apprentissage d'une langue 2 que ne peuvent l'être les départements de LEA. En déterminer les raisons est déjà une question de recherche et permettrait sans doute d'apporter une réponse mieux adaptée à la réalité du terrain. Il est possible d'avancer quelques hypothèses compte tenu de ce qui a été dit ci-dessus à propos du recrutement des enseignants. En effet, les enseignants de la filière ne sont pas recrutés nécessairement avec un profil LANSAD, ce qui a des conséquences considérables sur les contenus d'enseignements.

17 Les contenus sont parfois déterminés par la spécialité de l'enseignant plus que par les objectifs de la filière. On a ainsi vu quelques aberrations notables comme un cours sur le fantastique, genre littéraire, dispensé à des étudiants en LEA, mention affaires et commerce d'une université parisienne. Mais dans l'ensemble les effets sont plus pernicieux. Ainsi, dans certaines universités, on propose aux étudiants un cours de civilisation « ajusté » à leurs « capacités ", une sorte de cours de « civilisation pour nonspécialistes ", au lieu de réfléchir aux besoins réels des étudiants en ce qui concerne les acquisitions culturelles. On retrouve le même problème en traduction, où les références qui ont cours en LLCE sont appliquées directement en LEA, sans ajustements suffisants. Or tout différencie les deux filières : le niveau et le domaine de spécialisation en langue, les objectifs professionnels, la nature de la langue à acquérir, le rapport entre les heures de cours et le travail personnel. Les contenus ne peuvent de ce fait être semblables.

Pour un enseignement efficace en LEA, il serait souhaitable de réfléchir à des contenus adaptés aux objectifs de la filière qui se veut professionnalisante. Un programme convaincant et rassurant pour les étudiants ne devrait-il pas refléter une harmonie entre l'intitulé et les descriptifs et l'organisation des cours et leur contenu? C'est encore loin d'être toujours le cas.

19 À l'université de Nantes, la réflexion n'a pas suscité de révolution, mais quelques évolutions dans les approches et les contenus d'enseignement. L'étude de la langue, en anglais, est axée dès la première année sur la langue de l'entreprise en général, pour se spécialiser progressivement sur les genres et les types de discours propres au commerce international, ce qui n'était pas toujours le cas avant 2000. La traduction dans une langue et dans l'autre ne se limite plus à une traduction de texte de type universitaire, mais inclut progressivement le compte rendu, la revue de presse et la transposition (pour un site Internet, par exemple). L'étude de la langue s'organise autour de macro tâches, qui favorisent les interactions et l'apprentissage incident, impliquent les étudiants, et sont en cohérence avec les tâches attendues en entreprise. Cette approche nous ramène au cœur de la problématique de LEA qui concerne les problèmes liés à l'articulation théorie/ pratique. En effet, dans le monde du travail, on est dans la culture, comme ailleurs, mais l'urgence de comprendre s'y fait sentir de façon plus cruciale encore. Dans ce contexte, les acteurs manipulent un contenu professionnel avec une responsabilité sur le cours des événements. La question est de savoir si cela peut être enseigné ou s'il est tout au plus possible de sensibiliser les étudiants autour de tâches. 
Ce sont là quelques frémissements, à un petit niveau, en l'absence de moyens sérieux permettant de mobiliser des ressources humaines pour une recherche autour d'une équipe solide et motivée. Bien entendu, il serait nécessaire d'aller de l'avant et d'approfondir ces travaux dans plusieurs directions.

\section{Les dispositifs}

Le troisième volet du triptyque concerne les dispositifs mis en place. Là encore, on ne peut que constater l'inefficacité du système. L'université propose une organisation en CM, TD, TP. Ceci permet d'avancer qu'il ne semble pas y avoir dans ce domaine - qui demeure relativement auto-référencé - de questionnement sur les dispositifs. Or l'étude longitudinale mentionnée plus haut - sur trois années en M1 LEA à Nantes - fait apparaitre que le cours magistral pose problème. Ceci n'est pas une surprise au vu des théories didactiques : en effet, il est difficile de construire un savoir dans une posture transmissive. Pour vérifier l'hypothèse qu'il s'agit bien de l'entité par elle-même, l'enquête va être élargie à l'ensemble de la formation à l'UFR de langues, en anglais et LEA. Les résultats permettront d'aller plus loin, et peut-être d'éviter des pis-aller tels que les cours magistraux en français sur la base de l'hypothèse non vérifiée que les étudiants ne suivent pas parce qu'ils n'ont pas de connaissances suffisantes en langue 2 pour comprendre le contenu du cours.

Voyons quelques exemples de recherche didactique mise en place pour tenter de concilier les structures existantes et les théories sur l'apprentissage. Il n'est pas toujours possible de modifier les maquettes, ni même de remplacer les cours magistraux par d'autres dispositifs, il est néanmoins possible de réfléchir à d'autres modalités. Les étudiants peuvent par exemple disposer d'articles contradictoires, voire d'un polycopié du cours en langue 2. Ceux-ci sont accompagnés d'une tâche individuelle/individualisée amenant réflexion et conceptualisation. Une expérience semble donner des résultats positifs. Au début $\mathrm{du} \mathrm{CM}$, on affiche une dizaine de questions destinées à focaliser l'écoute, par exemple :

E-mails

When can e-mails be used?

When should they be avoided?

Why are TLAs used? When must they be avoided?

Why should emoticons be avoided in business?

Why do words written in capital letters have a negative impact?

Faxes

Why is it impossible to fax a Bill of Lading?

What is the difference between an e-mail and a fax in legal cases?

Le cours qui suit consiste à mettre en commun les réponses à ces questions, élaborées à partir des documents distribués, et à en débattre. On propose ainsi un objectif qui focalise l'écoute. Les échanges autour des questions conduisent à une confrontation des représentations, à un conflit sociocognitif qui favorise l'acquisition. Lorsque le professeur fait ensuite la synthèse des contributions, il peut rassembler les différents points de vue, et l'écoute est alors plus ciblée pour les étudiants.

Une alternative serait de remplacer le cours magistral par une présentation générale des objectifs et des modalités de travail, puis par des permanences où les étudiants 
pourraient rencontrer le professeur aux différentes étapes d'avancement des tâches qu'ils auraient à réaliser.

Soulignons qu'il ne s'agit que d'un exemple et que, comme toute action, il reste à en mesurer les effets. Les différents aspects du travail en LEA mériteraient dans leur totalité d'être passés au crible de la recherche-action.

\section{Conclusion : sur la nécessité d'une recherche concertée}

Comme on le voit, l'ampleur de la tâche est considérable et ne peut être menée de manière isolée. La problématique fait intervenir non seulement la didactique, mais aussi la sociologie de l'éducation : la participation basse notée lors des enquêtes longitudinales pourrait bien être liée au phénomène de massification, et la massification de l'enseignement universitaire être en corrélation avec la notion de récipiendaire passif. La psychosociologie intervient dans la réaction aux cours, et l'économie de l'éducation peut nous aider à élaborer une réflexion sur les ajustements nécessaires par rapport aux réalités socio-économiques et aux problèmes de financement (Delamotte 1998). En langue, l'articulation langue/matières d'application/responsabilité sociale rattache cette filière au secteur LANSAD. En didactique, nous avons vu que l'enseignement transmissif semble être peu remis en cause, ce qui nous conduit à réfléchir en termes de théorisation de l'apprentissage. Une réflexion sur les dispositifs et leur intégration dans un panorama global incluant les objectifs et les contenus de la formation s'avère également nécessaire, au-delà de l'intérêt intellectuel des réflexions sur la langue de spécialité et la civilisation par exemple.

Le mode de recherche en didactique, la recherche-action, interdit d'élaborer un programme de recherche figé. Comment construire un programme de recherche alors qu'il est impossible de prévoir les réactions des participants, étudiants, enseignants, institutions? La spécificité d'une filière appelle une réflexion didactique pour la mise en place des enseignements. En effet, la mise en place de formations en langue de spécialité, en civilisation, en traduction ou autre ne peut être transférée d'un contexte à un autre sans cette réflexion, et sans recherche-action pour en mesurer les effets.

Nous espérons par ce travail, fédérer les énergies et susciter la création d'équipes désireuses de s'impliquer dans ces recherches. Nous avons besoin du soutien de collègues débutants à l'Université et souhaitons les motiver pour ce type de recherche didactique qui, tout en rendant leurs enseignements mieux adaptés au public de LEA, leur permettrait de valoriser leurs efforts par des perspectives de carrière universitaire.

BIBLIOGRAPHIE

Delamotte, E. 1998. Une introduction à la pensée économique en éducation. Paris : Presses Universitaires de France. 
Little, D. 2005. « Language policy, language curricula, language pedagogy: creating optimum conditions for LSP in European Universities », conférence plénière au colloque du GERAS, Toulouse.

Micheli, Marie-Lise \& Agnès Villadary. 1997-1998. « Les déterminants de l'échec en premier cycle, analyse qualitative ». Étude financée par le ministère de l'Éducation nationale de l'enseignement supérieur, de la recherche et de l'insertion professionnelle.

Morin, E. 2005. La Méthode 6 : l'éthique complexe. Paris : Seuil.

Narcy-Combes, J.-P. 2005. Didactique des langues et TIC : vers une recherche-action responsable. Paris : Ophrys.

Narcy-Combes, M.-F. 2003. « La communication interculturelle en anglais des affaires : transfert ou conflit d'interprétation?». ASp 39-40, 119-129.

Simondon, G. 1989. L'individuation psychique et collective. Paris : Aubier.

\section{NOTES}

1. Langues pour spécialistes d'autres disciplines.

2. Micheli, Marie-Lise \& Agnès Villadary(1997-1998). Première partie (1997) : Le point de vue des enseignants. Deuxième partie (1998) : Le point de vue des étudiants. L'enquête a concerné dix enseignants, trois administratifs, et vingt étudiants en difficulté, dont dix en LEA. Le travail effectué donne des pistes, mais une recherche d'envergure apporterait des informations mieux utilisables.

\section{AUTEUR}

\section{MARIE-FRANÇOISE NARCY-COMBES}

Université de Nantes.mf.narcycombes@wanadoo.fr 